\title{
A COMPARISON OF THREE DIMENSIONAL MAXIMUM REACH ESTIMATION TECHNIQUES*
}

\author{
Louis E. Boydstunt, Thomas J. Armstrong $\ddagger$ and Fred L. Bookstein§ \\ tDepartment of Industrial and Operations Engineering, The University of Michigan, $2274 \mathrm{G}$. G. Brown \\ Laboratory, Ann Arbor, MI 48109, USA \\ $\ddagger$ Department of Environmental and Industrial Health, The University of Michigan \\ $\S$ Center for Human Growth, The University of Michigan
}

\begin{abstract}
Spline, periodic spline and spherical harmonic maximum reach estimation procedures are compared and evaluated based on ease of usage, estimation error and bias. The comparison indicates that each method provides comparable estimates based on percent variance accounted for $\left(R^{2}\right)$ and standard error. Spherical harmonies, however, are less biased and provide accurate estimates of the maximum reach sphere at the north and south poles, whereas splines and periodic splines are substantially biased in these regions. Three dimensional computer graphic depictions of reach data for the sea ted operator illustrate these biases.
\end{abstract}

\section{INTRODUCTION}

The ability of operators to reach all necessary controls and hand holds is of obvious importance in the safe operation of man-machine systems. Measurements of maximum reach capability are regularly used by designers to locate handholds and controls in reachable locations. Maximum reach data are readily available to the practitioner in the form of graphical representations of maximum reach contours which may be directly compared with the reach requirements for a particular task (Fig. 1). The use of reach contours, however, is usually restricted to a small range of body postures and seat designs. Therefore, reach contours must be derived for a variety of postural and task conditions to be of practical use.

The derivation of reach contours would be facilitated by the development of efficient data analysis procedures. Currently, fifth, fiftieth and ninety-fifth percentiles are obtained from ranked maximum reach data and contours are derived from point by point plots or by regression estimates of maximum reach distances in horizontal or vertical planes (Kennedy, 1964, 1976, 1978; Chafee, 1969; Haslegrave, 1970; Rozier, 1977). These procedures are time consuming, hinder the derivation of reach contours for a variety of conditions, and preclude the development of general models for individual and population maximum reach estimation.

Several promising techniques are available for modelling maximum reach capabilities including splines, periodic splines and spherical harmonics. These techniques do not rely on visual or qualititative procedures, provide minimum variance estimates over the entire reach surface, and are readily adaptable for use in interactive computer aided job analysis pro-

* Received 22 May 1979; in revised form 19 October 1979. cedures. The purpose of this paper is to compare these three techniques with respect to reach analysis requirements.

\section{Spline regression}

The method of splines provides a polynomial estimation function and, in this respoct, is similar to standard polynomial regression techniques. However, spline analysis proceeds by dividing the range of the independent variable into a series of $k$ regions or intervals which are separated by 'knots' $\left(\lambda_{0}, \lambda_{1}, \ldots, \lambda_{k}\right.$, as shown in Fig. 2). This procedure allows the derivation of a low order polynomial in each region.

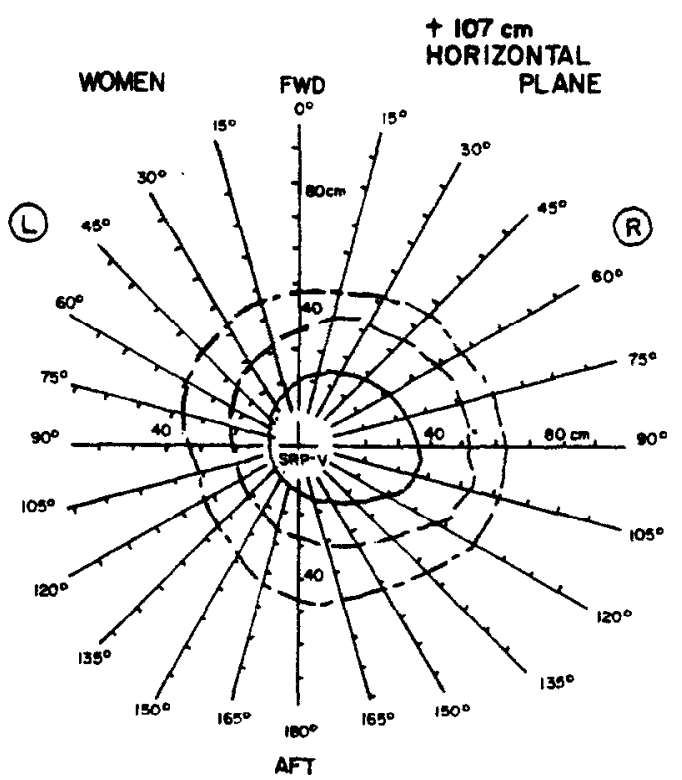

Fig. 1. Reach Contours for 5th, 50th, and 95 th percentile women in the sitting posture with shoulder restraints (Kennedy, 1976). 
$f(x)=f\left(1, x, x^{2}, x^{3}\right)$

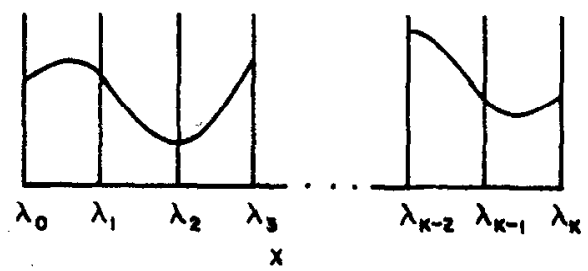

Fig. 2. Two-dimensional third order splines.

An accurate estimate may be obtained by decreasing the width of the intervals. In contrast, the accuracy of a standard polynomial regression may only be increased by increasing the polynomial order of the equation.

Spline analysis also insures that the estimation function is smooth as it crosses each of the knots. A spline function $f(x)$ which is a polynomial of order $m$ within each interval will be continuous up to and including the derivative of order $(m-1)$. For example, a cubic spline will be continuous at each knot approached from either the left or right side of the knot for position, slope and acceleration. Thus, for all values of $X$ within the range of interest:

$$
\begin{aligned}
& \lim _{\Delta x \rightarrow 0} f(X-\Delta X)=\lim _{\Delta X \rightarrow 0} f(X+\Delta X) \\
& \lim _{\Delta x \rightarrow 0} f^{\prime}(X-\Delta X)=\lim _{\Delta X \rightarrow 0} f^{\prime}(X+\Delta X)
\end{aligned}
$$

and

$$
\lim _{\Delta x \rightarrow 0} f^{\prime \prime}(X-\Delta X)=\lim _{\Delta x \rightarrow 0} f^{\prime \prime}(X+\Delta X)
$$

A one dimensional spline of polynomial order $m$ with $(k+1)$ knots $\left\{\lambda_{0}, \lambda_{1}, \ldots, \lambda_{k-1}, \lambda_{k}\right\}$ requires $(k+m)$ parameters. The estimation function for equally spaced knots is given by Prenter (1975):

$$
f(X)=\sum_{i=1}^{k+m} C_{i} B_{i}^{m}(X)
$$

where

$$
B_{i}^{m}(X)=h^{m} F^{m+1}\left(X-\lambda_{1}\right)^{m}
$$

and $h$ is the interval width between any adjacent pair of knots.

The forward difference is recursively defined by:

$$
F^{p+1}\left(X-\lambda_{i}\right)_{+}^{m}=\left\{\begin{array}{c}
F^{p}\left(X-\lambda_{i}\right)_{+}^{m}-F^{p}\left(X-\lambda_{i-1}\right)_{+}^{m} ; \\
p=1,2, \ldots, m \\
\left(X-\lambda_{i}\right)_{+}^{m}-\left(X-\lambda_{i-1}\right)_{+}^{m} ; \\
p=0
\end{array}\right.
$$

where

$$
\left(X-\lambda_{i}\right)_{+}^{m}=\left\{\begin{array}{c}
\left(X-\lambda_{i}\right)^{m} ;\left(X-\lambda_{i}\right)>0 \\
0 ; \text { otherwise }
\end{array}\right.
$$

Note that $m$ additional knots must be added to each end of the range of the independent variable (Fig. 3). Least squares procedures may be used to estimate the $(k+m)$ parameters.

The coefficients $C_{i}$ associated with each basis function $B_{l}^{m}(X)$ are 'local' since each basis function is nonzero across only $(m+1)$ adjacent intervals as shown in Fig. 2. Thus, coefficient $C_{l}$ contributes to the estimate only in the interval $\left\{\lambda_{1-m-1}, \ldots, \lambda_{i}\right\}$. In addition, parameter estimates $\hat{C}_{i}$ are primarily determined by data within this interval. The influence of data outside this interval becomes less important as the distance to the interval boundaries increases. The effect of outliers may, therefore, be localized by either judicious placement of knots or by utilizing a large number of equally spaced knots.

Spline analysis may be generalized to any order with unequally spaced knots in two dimensions through the use of tensor products of divided differences (Prenter, 1975) which yield the estimation function:

$$
f(X, Y)=\sum_{i=1}^{m+k} \sum_{j=1}^{n+l} C_{i j} D_{i}^{m+1}(X) D_{j}^{n+1}(Y)
$$

where:

$$
D_{i}^{m+1}(X)=\Delta^{m+1}\left(X-\lambda_{i}\right)^{m}
$$

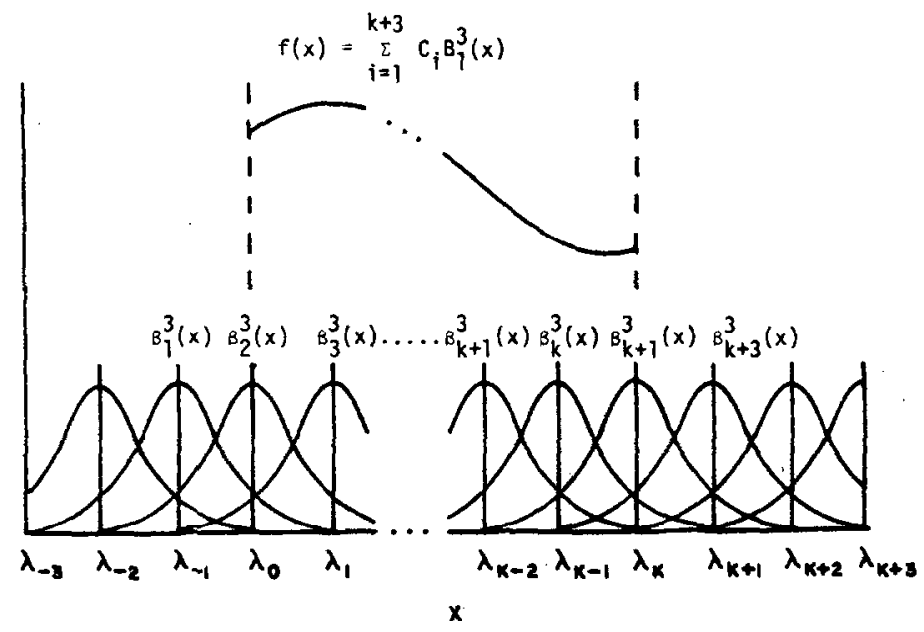

Fig. 3. Basis functions for third order splines with equally spaced knots. 
and

$$
D_{j}^{n+1}(Y)=\Delta^{n+1}\left(Y-\mu_{j}\right)_{+}^{n}
$$

Additional knots have been added to each dimension to give the following knot structure:

$$
\left\{\lambda_{-m}, \lambda_{-m+1}, \ldots, \lambda_{0}, \lambda_{1}, \ldots, \lambda_{k}, \lambda_{k+1}, \ldots, \lambda_{k+m}\right\}(
$$

and

$$
\left\{\mu_{-n}, \mu_{-n+1}, \ldots, \mu_{0}, \mu_{1}, \ldots, \mu_{l}, \mu_{l+1}, \ldots, \mu_{l+n}\right\}(5 b)
$$

where

$$
\lambda_{0}<X<\lambda_{k} \text { and } \mu_{0}<Y<\mu_{1}
$$

The divided difference is defined recursively by:

$\Delta i^{+1}\left(X-\lambda_{i}\right)_{+}^{m}$

$$
= \begin{cases}\frac{\Delta P\left(X-\lambda_{i}\right)_{+}^{m}-\Delta P-1}{\lambda_{i}-\lambda_{i}-P-1}\left(X-\lambda_{i-1}\right)_{+}^{m} & p=1,2, \ldots, m \\ \frac{\left(X-\lambda_{i}\right)_{+}^{m}-\left(X-\lambda_{i-1}\right)_{+}^{m}}{\lambda_{i}-\lambda_{i-1}} & p=0\end{cases}
$$

where:

$$
\left(X-\lambda_{i}\right)_{+}^{m}=\left\{\begin{array}{c}
\left(X-\lambda_{1}\right)^{m} ;\left(X-\lambda_{1}\right)>0 \\
0 ; \text { otherwise }
\end{array}\right.
$$

Divided differences in $Y$ are similarly defined. Standard least squares procedures may be used to estimate the spline parameters $C_{1 y}$ for many applications. Applications with a large number of knots or with closely spaced knots may require more complex procedures for evaluating the basis functions and for parameter estimation (DeBoor, 1972; Cox, 1972; Hays and Haliday, 1974; Dierckx, 1977).

Spline analysis of reach data in cartesian coordinates is not feasible since the maximum reach surface is doubly defined over the range of the independent variables (Fig. 4). Thus, reach distance ( $r$ ) is estimated in spherical coordinates as a function of latitude $(\phi)$ and longitude $(\theta)$. This procedure is based on the more reasonable assumption that the reach surface is 'star convex'. That is, each ray emitting from a center located within the reach sphere intersects the reach surface once and only once. Thus, the radius is assumed to be a single valued function of latitude and longitude.

\section{Periodic spline regression}

The analysis of reach contour data should presumably proceed under the assumption that the maximum reach surface is in some measure smooth and, therefore, without discontinuities on the reach surface. Spline analysis, as discussed above, insures that the estimation function is smooth at each of the longitudinal and latitudinal knots. However, spline analysis does not insure smoothness at the ends of the latitude and longitude ranges. Thus, there is a discontinuity at each pole of the reach surface, and a 'seam' of

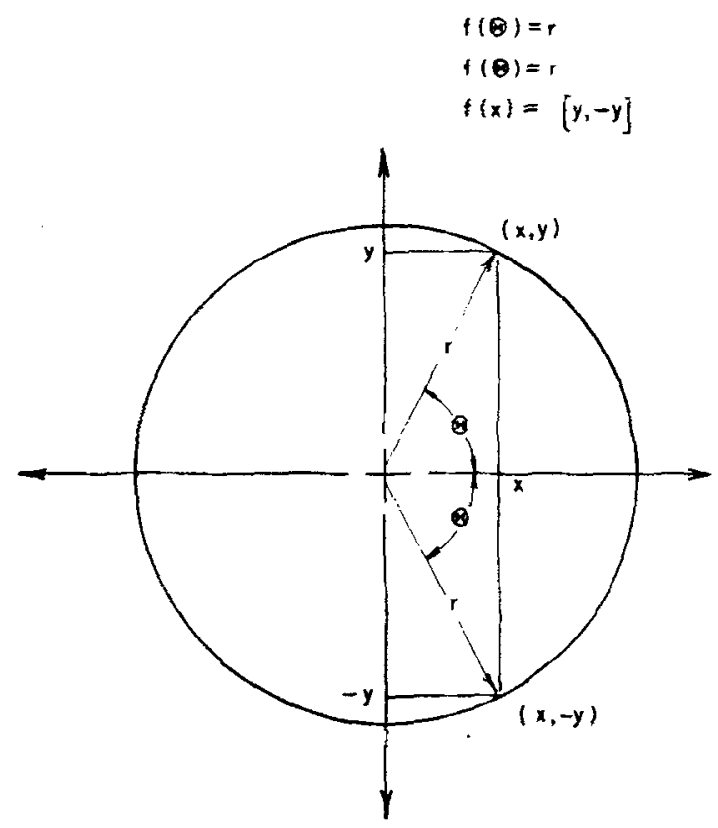

Fig. 4. Example of a double valued function in Cartesian coordinates which is single valued in polar coordinates.

discontinuities along the surface at $\theta= \pm 180^{\circ}$.

Periodic spline analysis resolves the latter problem by explicitly incorporating the periodicity of the estimation function along the longitudinal axis. The estimation function is given by :

$$
f(\theta, \phi)=\sum_{i=1}^{k} \sum_{j=1}^{n+1} C_{i j} D_{i}^{m+1}(\theta) D_{j}^{n+1}(\phi)
$$

where the longitudinal knots are constrained by $\lambda_{i+k}$ $=\lambda_{i}+180^{\circ}, k>m$. This insures that:

$$
f^{2}\left(180^{\circ}, \phi\right)=f^{3}\left(-180^{\circ}, \phi\right) ; s=0,1, \ldots, m-1
$$

The latitudinal divided differences $D_{j}^{n+1}(\phi)$ are equivalent to the basis functions defined for the nonperiodic splines in the previous section. The longitudinal constraints are easily implemented on a digital computer by transferring the non-zero elements from

$$
\left\{D_{k+1}^{m+1}(\theta), \ldots, D_{k+m}^{m+1}(\theta)\right\} \text { to }\left\{D_{i}^{m+1}(\theta), \ldots, D_{m}^{m+1}(\theta)\right\}
$$

respectively (see Fig. 5), prior to forming the tensor products which are required for the estimation functions. Otherwise the functions $\left\{D_{1}^{m+1}(\theta), \ldots\right.$, $\left.D_{k}^{m+1}(\theta)\right\}$ are defined the same as for non-periodic splines.

\section{Spherical harmonics}

The analysis of reach contours in spherical coordinates gives rise to two irregularities, as discussed in the previous section. The first, continuity of the estimation function at $f\left( \pm 180^{\circ}, \phi\right)$, was resolved through the use of periodic splines. The second and unresolved irregularity lies at the poles of the reach sphere which are completely specified at $\phi= \pm 90^{\circ}$ for all values of $\theta$. The reach estimation function should 


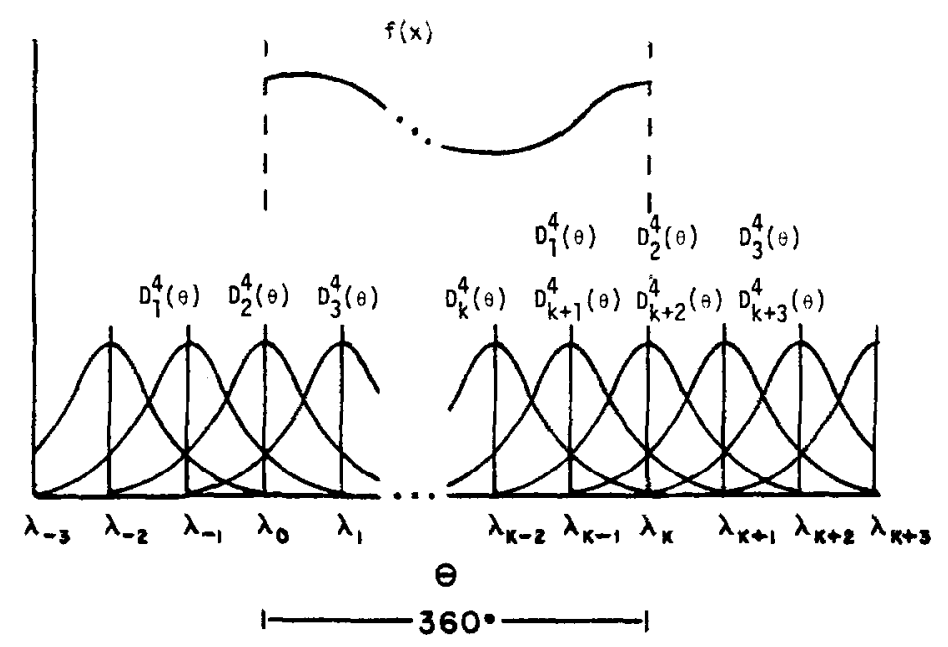

Fig. 5. Basis functions for third order periodic splines with equally spaced knots.

yield a single estimate for each pole which is independent of $\theta$. Thus, an estimation function is needed which is ; (1) periodic in $\theta$; and (2) independent of $\theta$ at the poles.

Spherical harmonics provide these properties by combining the periodic Fourier functions with Ferrers' associated Legendre functions (MacRobert, 1967). The basis functions are of the form:

$$
\begin{aligned}
& \left.\begin{array}{l}
\cos m \theta \\
\sin m \theta
\end{array}\right\} \sin ^{m} \phi P_{n}^{m}(\cos \phi) ; m=0,1, \ldots, n \\
& 0<\theta<2 \pi \\
& 0<\phi<\pi
\end{aligned}
$$

$P_{n}^{m}(\cos \phi)$ is the derivative of order $m$ of the Legendre polynomial of order $n$ (Table 1 ). The basis functions are clearly periodic in $\theta$ and are single valued at each pole. Note that the coordinate system is transposed so that $\phi$ now ranges from $0^{\circ}$ to $180^{\circ}$.

An estimation function which utilizes spherical harmonics is given by:

$$
\begin{array}{r}
f(\theta, \phi)=\sum_{i=0}^{n} \sum_{m=0}^{i}\left(B_{i m} \cos m \theta+C_{b m} \sin m \theta\right) \\
\sin ^{m} \phi P_{n}^{m}(\cos \phi)
\end{array}
$$

The parameters $C_{i m}$ and $B_{i m}$ may be estimated using standard least squares procedures.

Upon rotation of the coordinate system any spherical harmonic basis function is re-expressible as a linear combination of the original basis functions of the same order. Therefore, the components of variance associated with the series of orders (Table 1) are exactly invariant under rotation. In contrast, splines and periodic splines do not have this property. Thus, the quality of a spline estimate depends on the orientation of the coordinate system. The accuracy of both spherical harmonic and spline estimates is dependent on placement of the origin.

Spherical harmonic basis functions are orthogonal with respect to spherical area measure. The authors have found that the nonorthogonality among spherical harmonic basis functions is small even with missing data regions: the correlations among our basis func- tions average only 0.02 in absolute value for data placed at 15 degree intervals. However, efficiency of data collection procedures may be a consideration for spherical harmonics. For data collected at equally spaced degree intervals on the longitudinal and latitudinal axes, the horizontal spacing of the sample at latititudes $\pm 60^{\circ}$ is half the horizontal spacing at the equator corresponding to a doubled sample density. A better and cheaper sample would be based on a regular or semi-regular polyhedron, one whose vertices all have the same relation to the ensemble and thus are equivalent under symmetries of rather high order. For instance, the vertices of a truncated icosahedron comprise 60 sample directions each at a constant arcdistance from its three nearest neighbors (Holden, 1971, p. 43). The nonuniformity of this scheme will not be manifest in coefficients of order less than 5. Practical considerations, however, may dictate data placement.

\section{METHOD}

The establishment of generalized procedures for estimating individual and population reach capabilities would be facilitated by the establishment of a data base consisting of individual reach profiles. The data base should include model parameters, a summary of missing or unreachable areas and anthropometric measures for each individual. A simple model is advantageous since a generalized individual or population model, perhaps based on anthropometric measures, will be most easily derived from parsimonious individual models with identical structures or basis functions. This implies that spline knot locations should be the same for each individual. Equally spaced knots are selected for simplicity. This approach avoids a different optimal knot structure for each individual and work environment but may result in somewhat less accurate reach estimates. Spherical harmonics provide the desired model simplicity since only one set of basis functions are provided. Maximum reach data for an individual (Kennedy, 1976) was selected to compare spline, periodic spline and spherical har- 
monic analysis techniques. Each technique was limited to twenty-five parameters or less to provide a parsimonious model.

\section{RESULTS}

The original reach data (Fig. 6) show that missing data are confined to areas which are below and to the rear of the subject. The results of the analysis (Table 2) indicate that spherical harmonics account for a somewhat larger percentage of the variance when less than ten parameters are included in the estimation function. Estimation functions with sixteen or more parameters yield similar results for each of the techniques.

Graphical depictions of the estimation functions (Fig. 7) reveal substantial estimation biases at the north and south poles for spline and periodic spline analysis techniques. The seam to the rear of the subject which runs from the north pole to the south pole is not clearly visible for the spline analysis (Fig. 7a). However, the continuity of the periodic spline reach sphere (Fig. 7b) to the rear of the subject is clearly visible. A contour map of the spherical harmonic estimation function (Fig. 8) concisely represents the maximum reach data.

\section{DISCUSSION}

Splines, periodic splines and spherical harmonics give comparable estimates over the range of the data based on standard errors and multiple correlation coefficients. Each technique tends to be biased in regions to the rear and below the subject, which contain missing data, as expected. Periodic splines and spherical harmonics tend to give more plausible estimates in these regions since they take advantage of assumed regularities in the maximum reach surface.

Periodic splines yield biased estimates at the poles. This bias is not the result of unstable numerical procedures, but rather is due to a lack of constraints which would force continuity at the poles. Unfortunately, the constraints are not simple, as in the

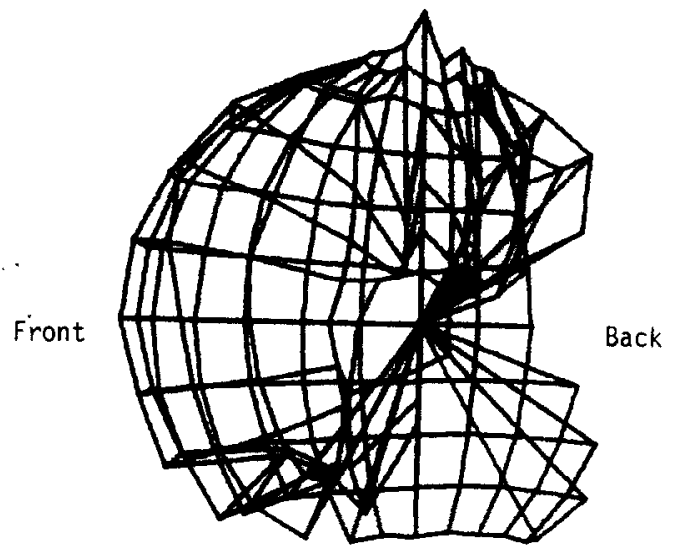

Fig. 6. Three-dimensional computer graphics depiction of an individual's maximum reach data (from Kennedy, 1976). The origin is $61 \mathrm{~cm}$ vertically above seat reference point. Missing data are coded as zeros. 
Table 2. Standard errors and multiple correlation coefficients for analyses of Kennedy (1976) reach data using (a) spline regression with equally spaced knots (b) periodic spline regression with equally spaced knots, and (c) spherical harmonic regression

\begin{tabular}{cccccc}
\hline Order & $\begin{array}{c}\text { Number of } \\
\text { knots in } \theta\end{array}$ & $\begin{array}{c}\text { Number of } \\
\text { knots in } \phi\end{array}$ & $\begin{array}{c}\text { Number of } \\
\text { parameters }\end{array}$ & $\boldsymbol{R}^{2}$ & $\begin{array}{c}\text { Standard } \\
\text { error }\end{array}$ \\
\hline 1 & 2 & 2 & 4 & 0.374 & 15.86 \\
1 & 3 & 3 & 9 & 0.689 & 11.32 \\
1 & 4 & 4 & 16 & 0.870 & 7.46 \\
2 & 2 & 2 & 9 & 0.769 & 9.77 \\
2 & 3 & 3 & 16 & 0.884 & 7.05 \\
2 & 4 & 4 & 25 & 0.893 & 6.94 \\
3 & 2 & 2 & 16 & 0.896 & 6.31 \\
3 & 3 & 3 & 25 & 0.909 & 6.85 \\
3 & 4 & 4 & 25 & 0.896 & 6.87 \\
\hline
\end{tabular}

(a)

\begin{tabular}{cccccc}
\hline Order & $\begin{array}{c}\text { Number of } \\
\text { knots in } \theta\end{array}$ & $\begin{array}{c}\text { Number of } \\
\text { knots in } \phi\end{array}$ & $\begin{array}{c}\text { Number of } \\
\text { parameters }\end{array}$ & $R^{2}$ & $\begin{array}{c}\text { Standard } \\
\text { error }\end{array}$ \\
\hline 1 & 3 & 4 & 12 & 0.837 & 8.26 \\
1 & 4 & 4 & 16 & 0.873 & 7.37 \\
1 & 5 & 4 & 20 & 0.885 & 7.11 \\
2 & 3 & 4 & 15 & 0.874 & 7.37 \\
2 & 4 & 4 & 20 & 0.886 & 6.87 \\
2 & 5 & 4 & 25 & 0.896 & 6.72 \\
3 & 4 & 4 & 24 & 0.902 & 6.72 \\
\hline
\end{tabular}

(b)

\begin{tabular}{cccc}
\hline Order & $\begin{array}{c}\text { Number of } \\
\text { parameters }\end{array}$ & $\boldsymbol{R}^{2}$ & $\begin{array}{c}\text { Standard } \\
\text { error }\end{array}$ \\
\hline 1 & 4 & 0.796 & 9.05 \\
2 & 9 & 0.836 & 8.23 \\
3 & 16 & 0.884 & 7.06 \\
4 & 25 & 0.895 & 6.90 \\
5 & 36 & 0.915 & 6.41 \\
\hline
\end{tabular}

(c)

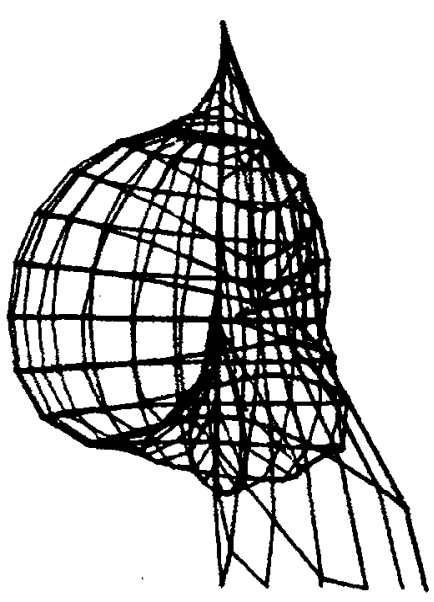

(a)

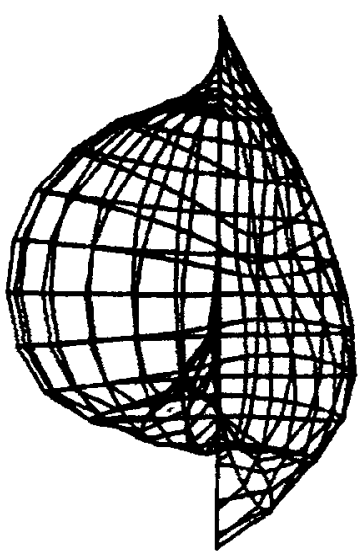

(b)

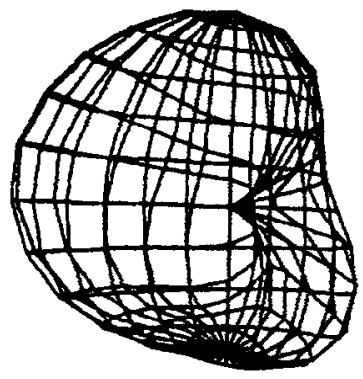

(c)

Fig. 7. Three-dimensional computer graphics depiction of an individual's maximum reach data (from Kennedy, 1976) using (a) spline regression with equally spaced knots (b) periodic spline regression with equally spaced knots, and (c) fourth order spherical harmonics. Each estimation function contains 25 


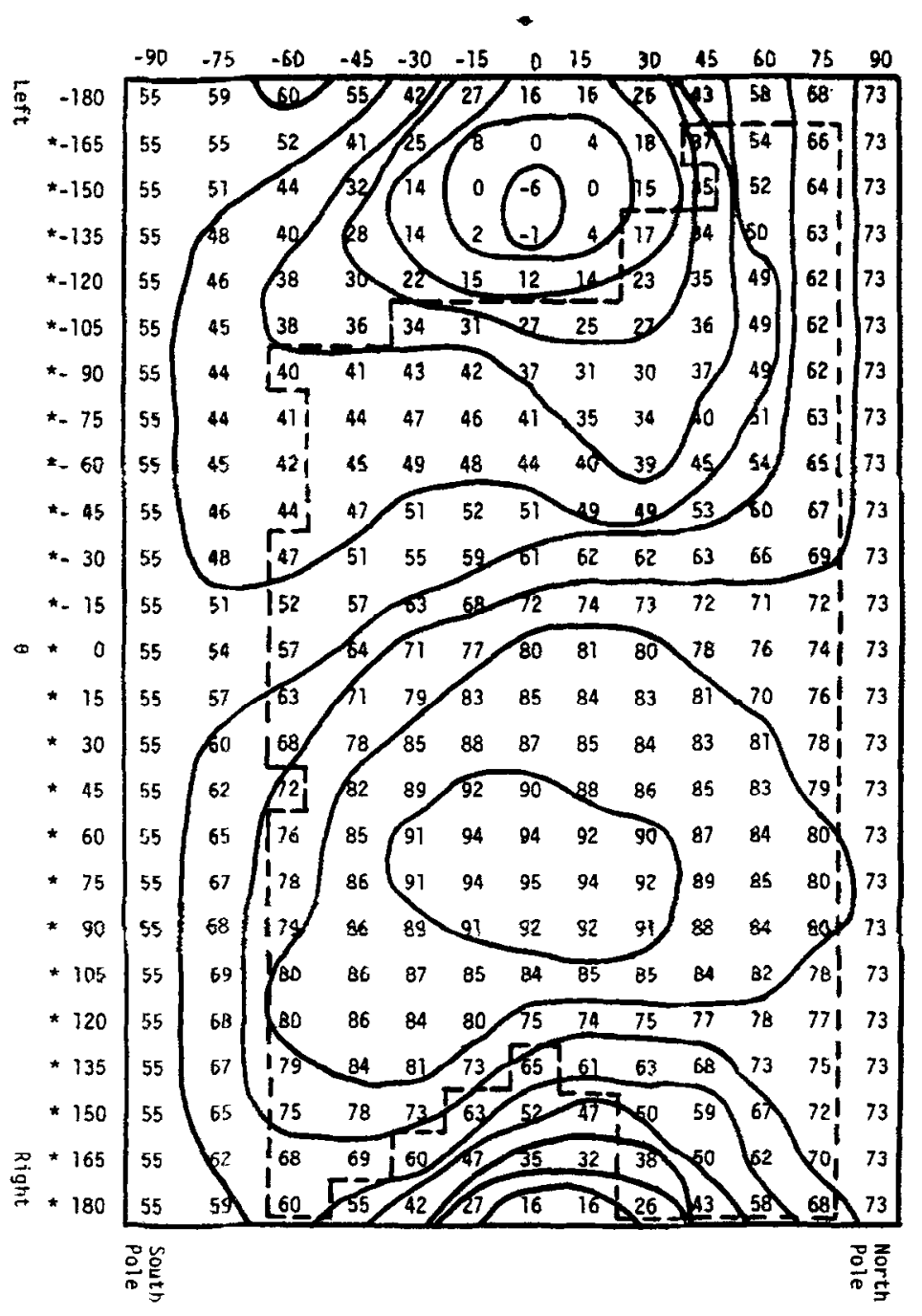

Fig. 8. Contour map of Kennedy (1976) reach data using fourth order spherical harmonics with 25 parameters.

periodic case. Additional constraints would have to be placed on non-adjacent basis functions at the north and south poles. For example, the slope of a latitudinal basis function at 0 degrees longitude would have to be equal in magnitude and opposite sign to the latitudinal basis function at 180 degrees longitude. Additional constraints would also be required for position and second derivative continuity. Thus, a great number of constraints would be required. Addition of these constraints disrupts the natural band structure of the splines.

Periodic spiines and spherical harmonics should give comparable estimates if more complete data were available. However, the spherical harmonics have the advantage of providing single reach estimates at the poles whereas periodic splines provide multiple estimates at the poles. Spherical harmonics are simpler to use since an underlying knot structure is not required. Also, the basis functions for spherical harmonics may be evaluated directly, whereas both the periodic and non-periodic splines require recursive computation of the basis functions. Spherical har- monics are therefore recommended based on ease of usage, estimation bias and estimation error.

The results of this study indicate that spherical harmonics provide a depiction of the maximum reach surface for an individual. Further research is needed to develop methods which will generalize spherical harmonics to provide population percentile data. In addition, a generalized methodology should provide the capability of determining the percentage of the population which will be capable of reaching several points in a work environment.

An important problem remains to be considered, namely obstructions in the environment. It is apparent that the procedures discussed in this paper will not form a general solution to the obstruction problem. The major difficulty is that the location of obstructions and other restrictions in the design space are specific to each application and may give rise to irregular maximum reach surfaces. Additional work is needed to identify major classes of obstructions which commonly occur and methods are needed for dealing with each class of ahstructions. 
The use of computer techniques for the analysis of three dimensional reach data reduces data storage requirements in comparison to standard pictoral representations (Fig. 1). Thus, reach parameters for a large number of individuals may be compactly stored in a data base. The ability to reproduce individual reach contours from parsimonious models is especially useful in the development of interactive computer graphics procedures for the analysis of alternative work station designs.

It should also be noted that the reach analysis techniques explored in this paper permit flexible data collection procedures. Thus, the researcher may: (1) gather data in the standard spherical coordinate format with reach distance as a function of latitude and longitude; (2) collect data in conveniently placed horizontal or vertical planes, or, (3) gather data at arbitrary points. Therefore, while equally spaced observations in spherical coordinates offers symmetry, other procedures will yield reliable results.

\section{REFERENCES}

Chaffee, J. W. (1969) Methods for Determining Driver Reach Capability. SAE Report 690105, New York.

Cox, M. G. (1972) The Numerical Evaluation of B-Splines. $J$. Inst. Maths. Applics. 10, 134-149.
De Boor, C. (1972) On Calculating with B-Splines. J. Approx. Theory 6, 50-62.

Dierckx, P. (1977) An Algorithm for Least Squares Fitting of Cubic Spline Surfaces to Functions on a Rectilinear Mesh Over a Rectangle. J. Comp. appl. Maths. 3, 113-129.

Haslegrave, C. M. (1970) Study of Reach to Car Controls While Restrained by a Lap and Diagonal Belt, MIRA Bulletin No. 1.

Hayes, J. G. and Halliday, J. (1974) The Least Squares Fitting of Cubic Spline Surfaces to General Data Sets, J. Inst. Maths. Applics. 14, 89-103.

Holden, A. (1971) Shapes, Space and Symmetry. Columbia University Press, New York, p. 43.

Kennedy, K. W. (1964) Reach Capability of the USAF Population. AMRL-TR-64-59, Wright-Patterson Air Force Base, Ohio.

Kennedy, K. W. (1976) A Dissertation in Engineering Anthropometry. Goodwin Watson Institute for Research and Program Development, 930 Corry Street, Yellow Springs, Ohio.

Kennedy, K. W. (1978) Reach Capability of Men and Women: A Three Dimensional Analysis. Aerospace Medical Research Laboratory, Wright-Patterson Air Force Base, AMRL-TR-77-50, July.

MacRobert, T. M.(1967) Spherical Harmonics, Ed.: Sneddon, 3rd Edition, Pergamon.

Prenter, P. (1975) Splines and Variational Methods. Now York.

Rozier, C. K. (1977) Three Dimensional Work Space of the Ampute. Human Factors 19, 525-533. 